\title{
PERIODONTAL DISEASE IN ASSOCIATION WITH SYSTEMIC DISEASES IN THE DOG
}

\author{
Penlington, L., Faixová, Z. \\ Institute of Pathological Physiology \\ University of Veterinary Medicine and Pharmacy in Košice \\ Komenského 73, 04181 Košice \\ Slovakia
}

zita.faixova@uvlf.sk

\section{ABSTRACT}

Periodontal disease (PD) is the second most common disease affecting dogs in UK veterinary practices. Veterinary and human literature suggests that periodontal disease may be associated with bacteraemia and a chronic, systemic release of inflammatory mediators which produce direct or immune-mediated changes elsewhere in the body. Thirty canine periodontal patient's electronic medical histories were analysed for comorbidities. The findings were analysed overall to identify any possible associations. Seventy three percent of these dogs had comorbidities, most commonly haematopoietic, cardiovascular, musculoskeletal and hepatic systems were involved. The most prevalent comorbidities were: high liver enzymes, heart murmur, mitral valve disease, and monocytosis. Other interesting comorbidities were: endocarditis, neutrophilia, submandibular lymph node enlargement and arthritis. Periodontal disease patients had a higher prevalence of disease when compared to the disease prevalence data for UK pet dogs in general. Mitral valve disease was over 17 times more likely in the periodontal disease patients. Comorbidity disease prevalence also increased with more severe periodontal disease stages. This study supports an association between periodontal disease and systemic diseases in the dog. Further studies should focus on confirming a cause and effect relationship. Until then, these data may be useful for veterinarians to examine periodontal patients for concurrent diseases and can be used as a tool to promote dental disease prevention to pet owners.

Key words: comorbidity; dog; periodontitis; periodontal disease; systemic disease

\section{INTRODUCTION}

Periodontal disease (PD) is the second most frequently seen disease in dogs in a veterinary practice in the United Kingdom [17]. Eighty percent of dogs show signs of periodontal disease by three years of age [29] and the incidence of the disease increases considerably with advancing age, causing significant oral pain and suffering [22]. Pet owners 
are currently recommended to brush their dog's teeth once per day, although compliance to this oral hygiene recommendation is low $[12,15]$.

Periodontal disease may act as a source of bacteraemia and chronic inflammation causing a chronic systemic release of inflammatory mediators, bacterial and cellular byproducts which may lead to direct or immune-mediated changes elsewhere in the body.

There is evidence in both the human and veterinary literature of an association between periodontal disease and systemic diseases. In humans, 57 systemic conditions are being researched in connection to periodontal disease; most of which are the result of chronic inflammatory disease [14], including cardiovascular diseases such as atherosclerosis, stroke and coronary heart disease $[1,16$, ], endocarditis [8], rheumatoid arthritis $[2,4,5$,], diabetes $[6,26]$ and pregnancy complications such as preeclampsia $[3,21]$, low birth weight [9] and premature delivery [28].

Less literature is available on dogs, although, mitral valve disease, atherosclerosis [19], histologic changes in the myocardium [7], endocarditis [11, 25], chronic kidney disease [10, 24], hepatic pathology [19], diabetes [13, 18, 20] and meningoencephalomyelitis [27] have been reported.
The aim of this study was to select canine periodontal patients, grade the severity of the periodontal disease, identify concurrent systemic diseases in each patient and analyse the overall findings to identify potential associations.

\section{MATERIALS AND METHODS}

\section{Sample selection}

Gatehouse Veterinary Centre in Lavister, a small animal first opinion veterinary practice in Wales, UK, provided access to their canine periodontal patient medical histories. The 30 most recent periodontal patient dental examination patients were selected, and their electronic medical histories analysed for concurrent diseases. The sample included 30 dogs of 18 breeds, aged 2 to 16 years of which 13 were females and 17 males.

\section{Stage of periodontal disease}

The patient's dental charts were analysed to determine the level of severity of periodontal disease. Guidelines for staging were outlined on the dental charts to allow the veterinary surgeons to categorise each stage in the same way.

Table 1. Periodontal patient medical histories

\begin{tabular}{|c|c|c|c|c|c|c|}
\hline & Breed & Age in years & Gender & Neuter status & PD stage & Comorbidities \\
\hline 1 & Cross & 11 & Male & Neutered & 1 & Dermatitis \\
\hline 2 & Cavalier King Charles spaniel & 9 & Male & Neutered & 1 & $\begin{array}{l}\text { Severe mitral valve disease, } \\
\text { Chronic heart failure }\end{array}$ \\
\hline 3 & Cavalier King Charles spaniel & 9 & Male & Neutered & 1 & $\begin{array}{l}\text { Mitral valve disease, } \\
\text { Heart murmur, } \\
\text { Otitis externa, } \\
\text { Cranial cruciate ligament rupture }\end{array}$ \\
\hline 4 & Shetland sheep dog & 5 & Male & Neutered & 1 & Heart murmur \\
\hline 5 & Miniature Schnauzer & 8 & Male & Intact & 1 & None \\
\hline 6 & Border terrier & 5 & Male & Neutered & 1 & None \\
\hline 7 & Yorkshire terrier & 5 & Male & Neutered & 1 & None \\
\hline 8 & Terrier & 2 & Male & Intact & 1 & None \\
\hline 9 & Border terrier & 13 & Male & Neutered & 2 & None \\
\hline 10 & West Highland terrier & 10 & Female & Neutered & 2 & $\begin{array}{l}\text { High total protein, } \\
\text { High liver enzymes, } \\
\text { Arthritis of the stifle, } \\
\text { Carnassial abscess }\end{array}$ \\
\hline
\end{tabular}


Table 1. Continues

\begin{tabular}{|c|c|c|c|c|c|c|}
\hline & Breed & Age in years & Gender & Neuter status & PD stage & Comorbidities \\
\hline 11 & Cavalier King Charles spaniel & 10 & Male & Intact & 2 & Anal adenoma \\
\hline 12 & Miniature Poodle & 8 & Female & Neutered & 2 & $\begin{array}{l}\text { High bile acids, } \\
\text { Diffuse hepatic changes, } \\
\text { Liver disease, } \\
\text { Hepatic vascular dysplasia, } \\
\text { Splenic reactive lymphoid hyperplasia }\end{array}$ \\
\hline 13 & Dachshund & 8 & Female & Neutered & 2 & $\begin{array}{l}\text { Intervertebral disc disease, } \\
\text { Anal gland impaction, } \\
\text { Sebaceous cyst }\end{array}$ \\
\hline 14 & Cross & 8 & Female & Intact & 2 & $\begin{array}{l}\text { Erythrocytosis, } \\
\text { Reticulocytosis }\end{array}$ \\
\hline 15 & Bull terrier & 8 & Male & Neutered & 2 & Fibroadnexal hamartoma \\
\hline 16 & Coton de tulear & 7 & Male & Neutered & 2 & Lameness \\
\hline 17 & Jack Russel terrier & 9 & Female & Neutered & 2 & None \\
\hline 18 & Labradoodle & 4 & Male & Neutered & 2 & None \\
\hline 19 & Jack Russel terrier & 13 & Female & Neutered & 3 & None \\
\hline 20 & Jack Russel terrier & 16 & Male & Neutered & 3 & $\begin{array}{l}\text { High liver enzymes, } \\
\text { High total protein, } \\
\text { Osteoarthritis of the elbow }\end{array}$ \\
\hline 21 & Cross & 15 & Female & Intact & 3 & $\begin{array}{l}\text { High liver enzymes, } \\
\text { Squamous cell carcinoma under tongue }\end{array}$ \\
\hline 22 & Jack Russel terrier & 14 & Male & Neutered & 3 & Erythrocytosis \\
\hline 23 & Lhasa apso & 14 & Female & Neutered & 3 & $\begin{array}{l}\text { High urea, } \\
\text { Thrombocytosis, } \\
\text { Cystitis, }\end{array}$ \\
\hline 24 & West Highland terrier & 12 & Male & Neutered & 3 & $\begin{array}{l}\text { Monocytosis, } \\
\text { Neutrophilia, } \\
\text { Lameness, } \\
\text { Thrombocytosis }\end{array}$ \\
\hline 25 & Bichon-Frise & 11 & Female & Neutered & 3 & $\begin{array}{l}\text { Heart murmur, } \\
\text { High liver enzymes, } \\
\text { Lymphopenia, } \\
\text { Lameness, } \\
\text { Thrombocytosis }\end{array}$ \\
\hline 26 & Miniature poodle & 11 & Female & Neutered & 3 & $\begin{array}{l}\text { Mitral valve disease, } \\
\text { Endocarditis with valvular and septal lesions, } \\
\text { Heart murmur, } \\
\text { Monocytosis, } \\
\text { Submandibular lymph node enlargement }\end{array}$ \\
\hline 27 & Cocker Spaniel & 10 & Female & Intact & 3 & $\begin{array}{l}\text { Arthritis of the elbow, } \\
\text { Monocytosis }\end{array}$ \\
\hline 28 & Yorkshire terrier & 10 & Female & Neutered & 3 & $\begin{array}{l}\text { High liver enzymes, } \\
\text { Erythrocytosis, } \\
\text { Leukopenia }\end{array}$ \\
\hline 29 & Jack Russel terrier & 8 & Female & Neutered & 3 & Reticulocytosis \\
\hline 30 & Chihuahua & 6 & Male & Neutered & 3 & $\begin{array}{l}\text { Heart murmur, } \\
\text { Intervertebral disc disease }\end{array}$ \\
\hline
\end{tabular}


Table 2. The most common organ systems affected in PD patients and their corresponding comorbidities

\begin{tabular}{|c|c|c|}
\hline $\begin{array}{l}\text { Organ } \\
\text { System }\end{array}$ & Comorbidity & $\begin{array}{c}\text { Number of } \\
\text { PD patients affected }\end{array}$ \\
\hline \multirow{7}{*}{ Haematopoietic } & Monocytosis & 3 \\
\hline & Thrombocytosis & 3 \\
\hline & Erythrocytosis & 3 \\
\hline & Reticulocytosis & 2 \\
\hline & Leukopenia & 1 \\
\hline & Lymphopenia & 1 \\
\hline & Neutrophilia & 1 \\
\hline & & Total: 14 \\
\hline \multirow{4}{*}{ Cardiovascular } & Heart murmur & 5 \\
\hline & Mitral valve disease & 3 \\
\hline & Chronic heart failure & 1 \\
\hline & Endocarditis & 1 \\
\hline & & Total: 10 \\
\hline \multirow{5}{*}{ Musculoskeletal } & Lameness & 3 \\
\hline & Arthritis & 2 \\
\hline & Intervertebral disc disease & 2 \\
\hline & Cruciate rupture & 1 \\
\hline & Osteoarthritis & 1 \\
\hline & & Total: 9 \\
\hline \multirow{4}{*}{ Hepatic } & High liver enzymes in blood & 5 \\
\hline & Hepatic vascular dysplasia & 1 \\
\hline & High bile acids in blood & 1 \\
\hline & Liver disease & 1 \\
\hline & & Total: 8 \\
\hline \multirow{3}{*}{ Urinary } & High total protein in the blood & 2 \\
\hline & Cystitis & 1 \\
\hline & Urea in the blood & 1 \\
\hline & & Total: 4 \\
\hline \multirow{3}{*}{ Tumour } & Anal adenoma & 1 \\
\hline & Fibroadnexal hamartoma & 1 \\
\hline & Squamous cell carcinoma under tongue & 1 \\
\hline & & Total: 3 \\
\hline \multirow{2}{*}{ Glandular } & Anal gland impaction & 1 \\
\hline & Sebaceous cyst & 1 \\
\hline & & Total: 2 \\
\hline \multirow{2}{*}{ Lymphoid } & Splenic reactive lymphoid hyperplasia & 1 \\
\hline & Submandibular lymph node enlargement & 1 \\
\hline & & Total: 2 \\
\hline \multirow{2}{*}{ Dermatologic } & Dermatitis & 1 \\
\hline & Otitis externa & 1 \\
\hline & & Total: 2 \\
\hline \multirow[t]{2}{*}{ Dental } & Carnassial abscess & 1 \\
\hline & & Total: 1 \\
\hline
\end{tabular}


Stage 1 was characterised by marginal gingivitis, involving a red line with some oedema, Stage 2 was characterised by swelling and some bleeding and stage 3 was characterised by severe inflammation and spontaneous bleeding [23].

\section{Medical history analysis}

Using the electronic medical histories, haematology and biochemistry test results, pathology laboratory reports, dental records and consultation and surgery notes were analysed to identify comorbidities. This was facilitated by the practice recommending pre-anaesthetic blood tests for patients before dental examinations.

\section{RESULTS AND DISCUSSION}

The periodontal disease patient sample involved a wide range of breeds, ages, genders and periodontal disease stages. From the sample, $73 \%$ of the periodontal patients were found to have one or more comorbidities (Table 1).

There were common associations in the periodontal patient samples. Certain organ systems were affected more frequently than others. The haematopoietic and cardiovascular systems were the most prevalent systems affected (Table 2) and included heart murmur, mitral valve disease, chronic heart failure, endocarditis and monocytosis amongst other comorbidities.

Table 3. Top 10 most common comorbidities in periodontal patients

\begin{tabular}{cc}
\hline $\begin{array}{c}\text { Top 10 most common } \\
\text { Comorbidities }\end{array}$ & $\begin{array}{c}\text { Number } \\
\text { of patients affected }\end{array}$ \\
\hline High liver enzymes & 5 \\
Murmur & 5 \\
Mitral valve disease & 3 \\
Monocytosis & 3 \\
Lameness & 3 \\
Thrombocytosis & 3 \\
Erythrocytosis & 3 \\
Arthritis & 2 \\
Intervertebral disc disease & 2 \\
Reticulocytosis & 2 \\
\hline
\end{tabular}

The most common comorbidities were: high liver enzymes, heart murmur, mitral valve disease, monocytosis and lameness (Table 3). Other interesting concurrent diseases included: neutrophilia, submandibular lymph node enlargement and arthritis. These findings potentially reflect chronic, systemic inflammation.

There was a significantly higher disease prevalence in periodontal disease patients in the study when compared to UK pet dog data [17] (Table 4). In periodontal patients, mitral valve disease was over 17 times more likely, heart disease generally was over 6 times more likely and heart murmur was over 4 times more likely. This suggests that periodontal disease could be an important factor in the development of systemic diseases. The comorbidity prevalence increased with more severe periodontal disease.

Fifty percent of patients with stage 1 periodontal disease had comorbidities, $70 \%$ of patients with stage 2

Table 4. Disease prevalence [\%] in the UK dog population compared to periodontal patients

\begin{tabular}{lcc}
\hline Comorbidity & $\begin{array}{c}\text { Disease prevalence } \\
\text { data for UK pet dogs } \\
{[\mathbf{1 7 ]}}\end{array}$ & $\begin{array}{c}\text { Disease prevalence } \\
\text { in periodontal } \\
\text { disease patients }\end{array}$ \\
\hline Heart murmur & 3.94 & 16.7 \\
Heart disease & 0.88 & 6.7 \\
$\begin{array}{l}\text { Mitral valve } \\
\text { disease }\end{array}$ & 0.57 & 10 \\
Lameness & 1.7 & 10 \\
Arthritis & 2.83 & 6.7 \\
Hepatopathy & 0.49 & 3.33 \\
\hline
\end{tabular}

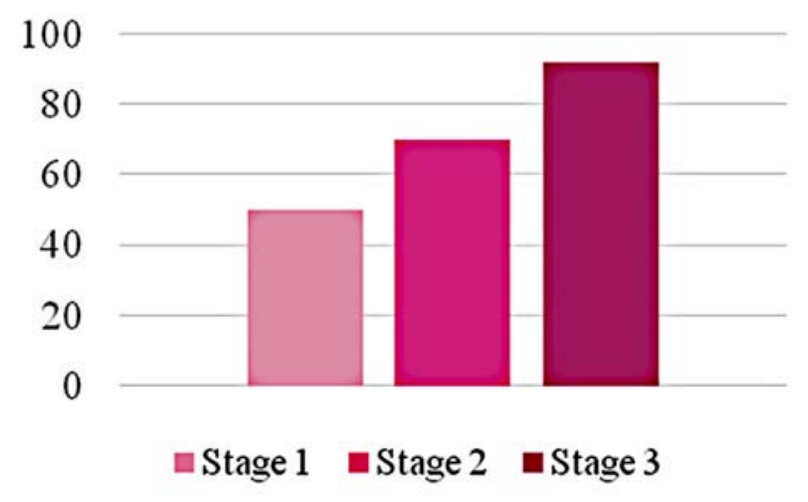

Fig. 1. Percentage of patients with comorbidities according to the periodontal disease severity 
had comorbidities and $92 \%$ of stage 3 had comorbidities (Fig. 1). This suggests that the more severe the periodontal disease becomes, the greater the chance of systemic disease developing.

\section{DISCUSSION}

The findings support an association between periodontal disease and systemic diseases in the dog. The data also support current literature which links canine periodontal disease to heart disease $[7,11,19,25]$ and hepatic pathology [19] and can be related to human associations such as: arthritis [2, 4, 5] and endocarditis [8].

This study suggests that periodontal disease could be an important factor in the development of systemic diseases. The identification of associations between periodontal disease and systemic diseases suggest that periodontal disease could be used as an early warning tool to detect concurrent diseases. Comorbidities could also be diagnosed and treated sooner resulting in improved welfare and longevity.

This study focused on an association as opposed to identifying a causal relationship. Future studies should work towards identifying a cause and effect relationship, for example by comparing microbiology samples from the periodontium and affected systemic organs. This would support the idea that treating and preventing periodontal disease may prevent and treat comorbidities such as endocarditis.

Until then, this data might be useful for veterinary surgeons to examine periodontal patients for concurrent diseases and can be used as a tool to promote dental hygiene recommendations to pet owners. It is currently recommended to brush a dog's teeth once per day using toothpaste especially made for dogs. Regular veterinary checkups are important to identify early signs of periodontal disease and veterinary treatment may be required to prevent its development.

\section{CONCLUSIONS}

Concurrent systemic diseases were identified in $73 \%$ of the periodontal patients sampled. The cardiovascular and haematopoietic systems were particularly affected. The most common comorbidities were high liver enzymes, heart murmur and mitral valve disease amongst others.
The comorbidity incidence increased with PD severity and there was significantly higher disease prevalences in the periodontal patient samples compared to the UK pet dog data. This study supports an association between periodontal disease and systemic diseases in the dog.

\section{ACKNOWLEDGEMENT}

We would like to thank Gatehouse Veterinary Centre in Lavister for participating in the study.

\section{REFERENCES}

1. Arabi, G., Eberhard, J., Reissmann, D., Heydecke, G., Seedorf, U., 2015: Interaction between periodontal disease and atherosclerotic vascular disease-Fact or fiction? Atherosclerosis, 241, 2, 555-560. Retrieved June 2019, from https://www. sciencedirect.com/science/article/pii/S0021915015010382. DOI: 10.1016/j.atherosclerosis.2015.04.819.

2. Beyer, K., Lie, S., Kjellevold, M., Dahl, L., Brun, J., Bolstad, A., 2018: Marine $\omega$-3, vitamin D levels, disease outcome and periodontal status in rheumatoid arthritis outpatients. Nutrition, 55-56, 116-124. Retrieved June 2019, from https://www.sciencedirect.com/science/article/pii/S08999007 18301849. DOI: 10.1016/j.nut.2018.03.054.

3. Boggess, K., Lieff, S., Murtha, A., Moss, K., Beck, J., Offenbacher, S., 2003: Maternal periodontal disease is associated with an increased risk for preeclampsia. Obstet. Gynecol., 101, 2, 227-231. Retrieved June 2019, from https://www. sciencedirect.com/science/article/pii/S0029784402023141. DOI: 10.1097/00006250-200302000-00006.

4. Calderaro, D., Corrêa, J., Ferreira, G., Barbosa, I., Martins, C., Silva, T., Teixeira, A., 2017: Influence of periodontal treatment on rheumatoid arthritis: a systematic review and meta-analysis. Rev. Bras. Rheumatol. (English Edition), 57, 3, 238-244. Retrieved June 2019, https://www.sciencedirect. com/science/article/pii/S2255502116301080. DOI: 10.1016/j. rbre.2016.11.011.

5. Cheng, Z., Meade, J., Mankia, K., Emery, P., Devine, D., 2017: Periodontal disease and periodontal bacteria as triggers for rheumatoid arthritis. Best Pract. Res. Clin. Rheumatol., 31, 1, 19-30. Retrieved June 2019, from https://www.sciencedirect.com/science/article/pii/S1521694217300116\#bib100. DOI: 10.1016/j.berh.2017.08.001Elsevier BV. 
6. Collin, H., Uusitupa, M., Niskanen, L., Kontturi-Närhi, V., Markkanen, H., Koivisto, Meurman, J., 1998: Periodontal findings in elderly patients with non-insulin dependent diabetes mellitus. J. Periodontol., 69, 9, 962-966. Retrieved June 2019, from https://onlinelibrary.wiley.com/doi/abs/10.1902/ jop.1998.69.9.962. DOI: 10.1902/jop.1998.69.9.962.

7. DeBowes, L., 1998: The effects of dental disease on systemic disease. Vet. Clin. North Am. Small Anim. Pract., 28, 5, 10571062. Retrieved June 2019, from https://www.sciencedirect. com/science/article/pii/S0195561698501027. DOI: 10. 1016/ s0195-5616(98)50102-7.

8. Dhotre, S., Jahagirdar, V., Suryawanshi, N., Daavane, M., Patil, R., Nagoba, B., 2018: Assessment of periodontitis and its role in viridans streptococcal bacteremia and infective endocarditis. Indian Heart J., 70, 2, 225-232. Retrieved June 2019, from https://www.sciencedirect.com/science/article/pii/ S0019483216301092. DOI: 10.1016/j.ihj.2017.06.019.

9. Gandhimadhi, D., Mythili, R., 2010: Periodontal infection as a risk factor for preterm low birth weight. J. Indian Soc. Periodontol., 14, 2, 114. Medknow. DOI: 10.4103/0972$124 \mathrm{x} .70832$.

10. Glickman, L., Glickman, N., Moore, G., Lund, E., Lantz., G, Pressler, B., 2011: Association between chronic azotemic kidney disease and the severity of periodontal disease in dogs. Prev. Vet. Med., 99, 2-4, 193-200. Retrieved June 2019, from https://www.sciencedirect.com/science/article/pii/ S0167587711000298. DOI: 10.1016/j.prevetmed.2011.01.011.

11. Glickman, L., Glickman, N., Moore, G., Goldstein, G., Lewis, H., 2009: Evaluation of the risk of endocarditis and other cardiovascular events on the basis of the severity of periodontal disease in dogs. J. Am. Vet. Med. Assoc., 234, 4, 486-494. Retrieved June 2019, from https://avmajournals.avma.org/doi/ abs/10.2460/javma.234.4.486. DOI: 10.2460/javma. 234.4.486.

12. Howell, T., Mornement, K., Bennett, P., 2016: Pet dog management practices among a representative sample of owners in Victoria, Australia. J. Vet. Behav., 12, 4-12. Retrieved June 2019, from https://www.sciencedirect.com/science/article/abs/pii/S1558787815002038. DOI: 10.1016/j.jveb.2015. 12.005. Elsevier BV.

13. Kuo, L., Polson, A., Kang, T., 2008: Associations between periodontal diseases and systemic diseases: A review of the interrelationships and interactions with diabetes, respiratory diseases, cardiovascular diseases and osteoporosis. Public Health, 122, 4, 417-433. Retrieved June 2019, from https://www.sciencedirect.com/science/article/pii/S0033350607002326. DOI: 10.1016/j.puhe.2007.07.004.
14. Loos, B., 2016: Periodontal medicine: work in progress! J. Clin. Periodontol., 43, 6, 470-471.

15. Miller, B., Harvey, C., 1994: Compliance with oral hygiene recommendations following periodontal treatment in clientowned dogs. J. Vet. Dent., 11, 1, 18-19. Retrieved June 2019, https://europepmc.org/abstract/med/7993583.

16. Mustapha, I., Debrey, S., Oladubu, M., Ugarte, R., 2007: Markers of systemic bacterial exposure in periodontal disease and cardiovascular disease risk: A systematic review and meta-analysis. J. Periodontol., 78, 12, 2289-2302. Retrieved June 2019, from https://onlinelibrary.wiley.com/doi/10.1902/ jop.2007.070140. DOI: 10.1902/jop.2007.070140.

17. O’Neill, G., Church, B., McGreevey, D., Thomson, C., Brodbelt, C., 2014: Prevalence of disorders recorded in dogs attending primary-care veterinary practices in England. PLoS ONE, 9, 3, e90501. Retrieved June 2019, from https://journals. plos.org/plosone/article?id=10.1371/journal.pone.0090501. DOI: 10.1371/journal.pone.0090501.

18. Oda, H., Mori, A., Saeki, K., Kurishima, M., Mimura, K., Nozawa, S., et al., 2011: Effect of periodontal treatment for glycemic control in dogs with diabetes mellitus. J. Pet Anim. Nutr., 14, 2, 76-83. Retrieved June, 2019 from JSTAGE database on the World Wide Web: https://www.jstage.jst.go.jp/ article/jpan/14/2/14_2_76/article. DOI: 10.11266/jpan.14.76.

19. Pavlica, Z., Petelin, M., Juntes, P., Eržen, D., Crossley, D., Skalerič, U., 2008: Periodontal disease burden and pathological changes in organs of dogs. J. Vet. Dent., 25, 2, 97-105. Retrieved June 2019, from http://journals.sagepub. com/doi/abs/10.1177/089875640802500210. DOI: $10.1177 /$ 089875640802500210 .

20. Pöppl, A., Muller, F., Queiroga, L., Oliveira, I., 2009: Insulin Resistance Due to Periodontal disease in an old diabetic female poodle. In World Small Animal Veterinary Association World Congress Proceedings, Rio Grande do Sul, Brazil, Mundo Animal Veterinary Hospital. Retrieved June 2019, from https://www.vin.com/apputil/content/defaultadv1.aspx?pId= $11290 \&$ meta $=$ generic\&catId $=33326 \&$ id $=4252831$ \&ind $=302$ \&objTypeID=17.

21. Rai, B., Kaur, J., Kharb, S., 2008: Pregnancy gingivitis and periodontitis and its systemic effect. The Internet Journal of Dental Science, 6, 2. Retrieved June 2019, from http://ispub. com/IJDS/6/2/5532.

22. Riggio, M., Lennon, A., Taylor, D., Bennett, D., 2011: Molecular identification of bacteria associated with canine periodontal disease. Vet. Microbiol., 150, 3-4, 394-400. Retrieved June 2019, from https://www.sciencedirect.com/sci- 
ence/ article/pii/S0378113511001398. DOI 10.1016/j.vetmic. 2011.03.001.

23. RVC.ac.uk., 2002: Preventative Veterinary Dentistry. Retrieved August 2019, from https://www.rvc.ac.uk/review/ dentistry/Shared_Media/pdfs/Prevent_print.pdf.

24. Scannapieco, F., Panesar, M., 2008: Periodontitis and chronic kidney disease. J. Periodontol., 9, 79, 1617-1619. DOI 10.1902/jop.2008.080313.

25. Semedo-lemsaddek, T., Tavares, M., Braz, B., Tavares, L., Oliveira, M., 2016: Enterococcal infective endocarditis following periodontal disease in dogs. PLoS ONE, 11, 1, e0146860. Retrieved June 2019, from https://journals.plos. org/plosone/article?id=10.1371/journal.pone.0146860. DOI: 10.1371/journal.pone.0146860.

26. Taylor, G., Burt, B., Becker, M., Genco, R., Shlossman, M., Knowler, W., Pettitt, D., 1996: Severe periodontitis and risk for poor glycemic control in patients with non-insulin-dependent diabetes mellitus. J. Periodontol., 67, 10s, 1085-1093. Retrieved June 2019, from https://onlinelibrary.wiley.com/doi/ abs/10.1902/jop.1996.67.10s.1085. DOI: 10.1902/jop.1996.67. 10s.1085.
27. Tun, A., Benedicenti, L., Galban, E., 2018: Pasteurella Multocida meningoencephalomyelitis in a dog secondary to severe periodontal disease. Clin. Case Rep., 6, 6, 1137-1141. Retrieved June 2019, from https://onlinelibrary.wiley.com/ doi/10.1002/ccr3.1561. DOI: 10.1002/ccr3.1561.

28. Wener, M., Lavigne, S., 2004: Can periodontal disease lead to premature delivery? AWHONN Lifelines, 8, 5, 422-431. Retrieved June 2019, from https://www.sciencedirect.com/science/article/pii/S1091592315311845. DOI: 10.1177/1091592 304271624.

29. Whyte, A., Bonastre, C., Monteagudo, L., Les, F., Obon, J., Whyte, J., Tejedor, M., 2014: Canine stage 1 periodontal disease: a latent pathology. Vet. J., 201, 1, 118-120. Retrieved June 2019, from https://www.sciencedirect.com/science/article/pii/s1090023314001890. DOI: 10.1016/j.tvjl.2014.05.005.

Received July 2, 2019

Accepted September 6, 2019 